\title{
DIELECTRIC AND THERMODYNAMIC PROPERTIES OF 2-AMINOPROPANE AND 2-ETHOXYETHANOL IN DIFFERENT NON POLAR LIQUIDS AT MICROWAVE FREQUENCY
}

\author{
N. S. RAMTEKE \\ Department of Physics, Dr. Ambedkar College, Chandrapur-442401, Maharashtra, India
}

\section{ABSTRACT}

Dielectric constant, dielectric losses of 2-Aminopropanc and 2-Ethoxyethanol in dilute solutions in different non polar media, viz., cyclohexane, benzene and heptane at $9.8 \mathrm{GHz}$ and at different temperatures have been determined. Dielectric data of the solutions have been utilized to determine microscopicrelaxation times and dipole moments of the systems. Temperature dependent relaxation times of the dilute solutions and viscosities of the solvent are utilized in the calculations of the thermodynamic energy parameters for the activated state of the system.

KEYWORDS: Dielectric constant, Dielectric Loss, Relaxation time, Dipole moment, Microwave absorption, Thermodynamic energy parameters

Received: Jan 14, 2021; Accepted: Feb 05, 2021; Published: Feb 24, 2021; Paper Id.: IJPRJUN20211

\section{INTRODUCTION}

Polar molecules have asymmetric distribution of positive and negative charges which on interaction with the microwave radiation contributes to dielectric permittivity. Dielectric measurements of polar molecule in dilute solutions of non-polar solvent in the microwave region is helpful in gaining more information about the polar molecule, viz., size, structure and interacting ability to its surrounding medium¹. 2-Aminopropane and 2Ethoxyethanol ${ }^{2}$ are extended for their dielectric relaxation study in different nonpolar media. Dielectric relaxation is the non quantal rotational process of the polar molecule in non-polar medium or in its pure liquid state ${ }^{3}$. The present investigations report the microscopic ${ }^{4}$ values of relaxation time $(\tau)$, dipole moment $(\mu)$ and thermodynamic energy parameter ${ }^{3}$ of the systems in three different non polar media. However for 2-ethoxyethanol the measurements are made in two different non polar solvents. The relaxation parameters of the systems are compared with respect to different solvent media.

\section{EXPERIMENTAL}

The chemicals have been obtained commercially with a purity of $99 \%$ specification and they are further purified by distillation before their use. However, 2-aminopropane is used without any further purification. Dilute solutions of polar systems were prepared at room temperature and used immediately for their dielectric measurements. Dielectric constant $\left(\varepsilon^{\prime}\right)$ and loss $\left(\varepsilon^{\prime \prime}\right)$ have been determined at $\mathrm{x}$-band $(9.8 \mathrm{GHz})$ at different temperatures by using standing wave technique of Smyth ${ }^{5}$ described in an earlier paper $^{2}$. The accuracy of the measurements for $\left(\varepsilon^{\prime}\right)$ and $(\varepsilon ")$ was found to be within $+2 \%$ and $+5 \%$ respectively. Viscosities of the solvent at different temperatures have been determined by using calibrated Ostwald type viscometer. Relaxation time $(\tau)$ and dipole moment $(\mu)$ of the systems are determined by using the Gopala- Krishna method ${ }^{6}$ and thermodynamic energy parameters by using the 
Eyring's relations ${ }^{3}$.

\section{RESULTS AND DISCUSSIONS}

Table-1 reports the values of relaxation time $(\tau)$, dipole moment $(\mu)$ and thermodynamic energy parameters of the 2Aminopropane and 2-Ethoxyethanol in three different non polar solvents, viz. cyclohexane, benzene and heptane. However, 2-Ethoxyethanol is reported only in two solvents (viz. cyclohexane and benzene) Viscosity of the three solvents at different temperatures is also reported. $\tau=2.25 \mathrm{pS}$ is the larger relaxation time of 2-Aminopropane in benzene at $8^{\circ} \mathrm{C}$ compared to its relaxation time value at corresponding temperature $(\tau=0.86 \mathrm{pS})$ in cyclohexane; though the benzene has lower viscosity as compared to cyclohexane. This larger relaxation time of 2-Aminopropane in benzene medium may be due to the weak associative interactions between 2-Aminopropane and benzene molecule (assuming solute-solute interactions in dilute solutions are absent). Free energy of activation for 2-Aminopropane in benzene is larger compared to its free energy of activation in cyclohexane which supports the assumption for weak associative interaction between them. However, the values of relaxation times and free energy of activation of 2-Aminopropane in heptane are found to be smaller compared to those values in benzene and cyclohexane. This may be interpreted as 2-Aminopropane and heptane molecules show non associative nature in their solution state. In fact viscosity of the heptane is smaller compared to benzene and cyclohexane and it may be the second reason for the smaller values of relaxation times of 2-Aminopropane in heptane.

The dielectric relaxation parameters of 2-Ethoxyethanol in benzene and cyclohexane media indicate that2Ethoxyethanol and benzene molecules behave as associative type of interactions in their solution state. The dipole moment values of the systems vary from 10 to $20 \%$ in different non polar media. It is difficult to say that this change is because of solvent effect, because experimental error on dipole moment is of the same order. The dipole moment values of the systems in benzene solution agree with the available literature value ${ }^{7}$.

The negative and positive values of entropy for dielectric relaxation and viscous flow process arearbitrary ${ }^{8}$. From Table-1, it is found that the free energies of activation for viscous flow are always larger than the free energies of activation for dielectric relaxation. This indicates that the viscous flow process involves both rotational and translation motion whereas dielectric relaxation involves only rotationalmotion ${ }^{3}$.

Table 1:Viscosity, Dielectric Relaxation and Thermodynamic Properties of Polar Solute at Different Temperatures in Different Non-Polar Solvent

\begin{tabular}{|c|c|c|c|c|c|c|c|c|c|}
\hline \multirow{2}{*}{$\begin{array}{c}\text { Polar } \\
\text { solute } \\
\text { Solvent, } \\
\text { Temp }\left({ }^{\circ} \mathrm{C}\right)\end{array}$} & \multirow{2}{*}{$\begin{array}{c}\text { Viscosity } \\
\text { псp }\end{array}$} & \multirow{2}{*}{$\begin{array}{c}\begin{array}{c}\text { Relaxation } \\
\text { time }\end{array} \\
\tau p s\end{array}$} & \multirow{2}{*}{$\begin{array}{c}\begin{array}{c}\text { Dipole } \\
\text { moment }\end{array} \\
\mu \mathrm{D}\end{array}$} & \multicolumn{6}{|c|}{$\begin{array}{c}\text { Thermodynamic energy parameters } \\
\text { (kcal/mole degree) }\end{array}$} \\
\hline & & & & $\Delta \mathbf{H} \tau$ & $\Delta \mathbf{F} \tau$ & $\Delta \mathbf{S} \tau^{*}$ & $\Delta \mathbf{H \eta}$ & $\Delta F \eta$ & $\Delta \mathbf{S} \eta^{*}$ \\
\hline \multicolumn{10}{|c|}{ 2-Aminopropane in cyclohexane } \\
\hline 8 & 1.03 & 0.87 & 1.40 & 2.8 & 1.27 & 5.4 & 2.43 & 3.18 & \multirow[t]{3}{*}{-2.65} \\
\hline 16 & 0.99 & 0.70 & 1.40 & & 1.24 & & & 3.20 & \\
\hline 25 & 0.89 & 0.51 & 1.31 & & 1.18 & & & 3.23 & \\
\hline \multicolumn{10}{|l|}{ Benzene } \\
\hline 8 & 0.71 & 2.24 & 1.13 & 3.9 & 1.53 & 8.8 & 2.11 & 2.90 & \multirow[t]{3}{*}{-2.83} \\
\hline 16 & 0.68 & 1.75 & 1.10 & & 1.46 & & & 2.93 & \\
\hline 25 & 0.46 & 0.76 & 1.01 & & 1.25 & & & 3.03 & \\
\hline \multicolumn{10}{|l|}{ Heptane } \\
\hline 5 & 0.52 & 1.75 & 1.06 & 3.6 & 1.41 & 7.9 & 1.20 & 2.90 & \multirow[t]{2}{*}{-6.13} \\
\hline 15 & 0.49 & 0.95 & 1.00 & & 1.33 & & & 2.96 & \\
\hline
\end{tabular}


Non Polar Liquids at Microwave frequency

\begin{tabular}{|l|l|l|l|l|l|l|l|l|l|l|}
\hline 25 & 0.46 & 0.76 & 1.01 & & 1.25 & & \multicolumn{2}{l|}{3.03} & \\
\hline 2-Ethoxyehanol in cyclohexane & \multicolumn{5}{l|}{} \\
\hline 10 & 1.03 & 5.01 & 1.54 & 2.3 & 1.93 & 1.4 & 2.44 & 3.18 & -2.65 \\
\hline 20 & 0.94 & 4.08 & 1.49 & & 1.91 & & & 3.21 \\
\hline 30 & 0.83 & 3.33 & 1.50 & & 1.90 & & & 3.24 \\
\hline 40 & 074 & 3.00 & 1.60 & & 1.89 & & & 3.28 \\
\hline 50 & 0.65 & 2.58 & 1.63 & & 1.87 & & & 3.31 \\
\hline Benzene \\
\hline 10 & 0.73 & 5.58 & 2.02 & 2.0 & 1.93 & 0.4 & 2.11 & 2.91 & -2.83 \\
\hline 20 & 0.66 & 4.68 & 2.04 & & 1.93 & & & 2.94 \\
\hline 30 & 0.58 & 4.25 & $2.12 \mathrm{~b}$ & & 1.92 & & & 2.97 \\
\hline 40 & 0.55 & 3.92 & 2.11 & & 1.82 & & & 3.01 \\
\hline 50 & 0.48 & 2.58 & 2.24 & & 1.91 & & & 3.04 \\
\end{tabular}

a-ref 2. b-ref 7. $\Delta \mathrm{S} \tau^{*}$ and $\Delta \mathrm{S \eta} \eta^{*}$ in $\mathrm{kcal} / \mathrm{mole}$ degree

\section{CONCLUSIONS}

The large values of relaxation time and free energy of activation of 2-Aminopropane in benzene solution indicate that there may be weak associative interactions between 2-Aminopropane and benzene molecule. Comparing relaxation parameters of 2-Ethoxyethanol in cyclohexane with corresponding values in benzene, it is concluded that there is a weak association between 2-Ethoxyethanol and benzene molecule.

\section{ACKNOWLEDGEMENTS}

The author is indebted to Dr. B.M. Suryavanshi, Head, Department of Physics, Institute of Science, Nagpur for valuable guidance and suggestions. The author is grateful to UGC India for financial support under minor research project.

\section{REFERENCES}

1. N.E Hill, W. E. Vaughan, A.H. Price and M. Davice, Dielectric Properties and Molecular Behaviour, Van Nostrand Reinhold Co. London (1969).

2. M. V. K. Mehar \& V. Somashekarrao, “ International Journal of Metallurgical \& Materials Science and Engineering (IJMMSE), Vol. 8, Issue 2, pp. 1-4

3. R. K. Nagarch, "A New Fast Ag+ Ion Conducting Composite Electrolyte System: Dielectric Investigations “, International Journal of Applied and Natural Sciences (IJANS), Vol. 5, Issue 5,pp. 79-86

4. BM. Suryawanshi and S.C. Mehrotra, Indian J. Pure Appl. Phy., 29, 482 (1991).

5. Suribabu. Miriyala, B. V. Sanker Ram \& K. S. R. Anjaneyulu, "Fault Detection of Power Transformer by Using Wavelet Transforms", BEST: International Journal of Management, Information Technology and Engineering (BEST: IJMITE), Vol. 3 , Issue 10, pp. 111-116

6. N. Dinesh Kumar, S. Pramod \& CH Sravani, “Intelligent Irrigation System “, International Journal of Agricultural Science and Research (IJASR), Vol. 3 Issue 3, pp.23-30

7. E.S. Glaston, K.J. Laidier and H. Eyring, Theory of Rate Process, McGraw Hill Book Co., New York .p. 548(1941).

8. Reference 1, p. 63 .

9. W.M. Hestpm (Jr.), A.D. Franklin, E.J. Heennelly and C.P. Smyth, J. Am. Chem. Soc., 723443 (1950).

10. K.V. Gopala Krishna, Trans. Farad. Soc., 53, 767, (1957). 
11. A.L. McClellan, Tables of Electric Dipole Moments, W.H. Freeman \&amp; Co., London (1963).

12. F.H. Branin (Jr.) and C.P. Smyth, J. Chem. Phys., 20, 11212 (1952). 